\section{Fatores associados ao tempo de ocorrência das reações hansênicas numa coorte de 2008 a 2016 em Rondônia, Região Amazônica, Brasil}

\author{
Factors associated with timing of lepra reactions \\ in a cohort from 2008 to 2016 in Rondônia, \\ Amazon Region, Brazil
}

\section{Factores asociados al tiempo de ocurrencia de las reacciones hansénicas en una cohorte de 2008 a 2016 en Rondonia, Región Amazónica, Brasil}

Carmelita Ribeiro Filha Coriolano 1,2

Walter Ataalpa de Freitas Neto 3

Gerson Oliveira Penna 1,4

Mauro Niskier Sanchez 1

\title{
Resumo
}

O manejo clínico de pacientes com hanseníase apresenta um desafio particular que são as reações. O objetivo deste estudo de coorte não concorrente foi analisar o tempo e fatores associados à ocorrência da primeira reação $d u-$ rante e após o tratamento da poliquimioterapia $(P Q T)$. Avaliou-se 1.621 pacientes paucibacilares $(P B=8,9 \%)$ e multibacilares $(M B=91,1 \%)$ de 2008 a 2016 notificados no Sistema de Estados Reacionais em Hanseníase/Rondônia (SisReação/RO). Prevaleceu a ocorrência durante o tratamento da $P Q T=997$ $(61,5 \%)$, e $624(38,5 \%)$ somente após o PQT. A precocidade da reação, a partir do diagnóstico, foi analisada por meio de curvas de sobrevida de KaplanMeier e comparadas entre os grupos $P B$ e $M B$, usando o teste de log-rank de Mantel-Cox; e foram construídos modelos de regressão de Cox univariada e multivariada para identificar os fatores associados à ocorrência da reação (hazard ratio) e os correspondentes IC95\%. No modelo multivariado foram incluídas variáveis com valores de $p<0,2$ na análise univariada. Os PB desenvolveram reação de forma mais precoce do que os $M B$. Outras características associaram-se à reação em menor tempo: sexo feminino e baciloscopia negativa. No período agregado (durante e após a PQT), os pacientes $P B$ apresentaram risco $24 \%$ maior de reação do que os $M B$ e aqueles com baciloscopia negativa aumentaram este risco em $40 \%$ comparado à baciloscopia positiva. Durante e após a $P Q T$, os $P B$ apresentaram 1,3 e 1,6 vezes maior risco de ocorrência da reação dos pacientes MB. Dessa forma, recomendamos priorizar ações de vigilância para reações hansênicas durante e após a PQT como medidas de prevenção de incapacidades físicas e de melhoria na qualidade de vida das pessoas acometidas pela hanseníase.

Hanseníase; Vigilância Epidemiológica; Análise de Sobrevida

\author{
Correspondência \\ C. R. F. Coriolano \\ AOS 5, bloco D, apto. 101, Brasília, DF 70.660-054, Brasil. \\ carmel.ribeiro@gmail.com \\ 1 Universidade de Brasília, Brasília, Brasil. \\ 2 Ministério da Saúde, Brasília, Brasil. \\ 3 Universidade Federal do Vale do São Francisco, Petrolina, \\ Brasil. \\ ${ }^{4}$ Escola de Governo, Fundação Oswaldo Cruz, Brasília, Brasil.
}




\section{Introdução}

A hanseníase é uma doença dermatoneurológica, infecciosa, sistêmica ou localizada, debilitante causada por Mycobacterium leprae 1. Desde a introdução da poliquimioterapia (PQT) há cerca de quatro décadas, com a redução do tempo de tratamento, a prevalência da doença caiu, mas não impactou a transmissão da doença. Portanto, atualmente, muitos países ainda apresentam altas taxas de detecção 2 , sobretudo em áreas de maior vulnerabilidade social 3, havendo evidências robustas sobre os fatores socioeconômicos associados ao risco de adoecer 4 e de desenvolver incapacidades físicas 5 .

O Brasil ocupa a segunda posição entre os países com maior número de casos novos de hanseníase, onde também se concentra mais de $90 \%$ de todos os casos da doença nas Américas 6. O Norte está entre as regiões do país com maior carga da doença, sendo a porção da Amazônia Legal a mais acometida 7. O Estado de Rondônia, está nesse contexto de endemicidade, ficando na terceira colocação na taxa de detecção geral entre os estados da região, em 2019. Esse cenário coloca o estado, a região e o Brasil em posição de destaque desconfortável.

Uma das principais dificuldades no manejo clínico de pacientes com hanseníase é o desenvolvimento de reações, episódios agudos que ocorrem no curso da doença crônica e são fenômenos imunes inflamatórios, que podem causar incapacidades físicas irreversíveis 1 . Esses fenômenos podem ser localizados ou sistêmicos 8 , se expressam, em sua maioria, de forma sintomática; ocorrem antes, durante ou após o tratamento específico da doença 8,9,10,11 e guardam relação com a carga bacilar e da interação M. Leprae-hospedeiro ${ }^{12}$, necessitam de intervenção imediata $8,9,13$.

As principais reações hansênicas são: reação Tipo 1 ou reversa (RR) 1,9,14, com ou sem neurite 8,15, estando associada à resposta imune celular (do tipo Th1); e a reação Tipo 2 que está relacionada à ausência de resposta Th1 e relativa expressão Th2 (imunidade humoral), cuja manifestação clínica mais frequente é o eritema nodoso hansênico (ENH) 1,10,16,17,18, com ou sem neurite 8,15 . Considera-se ainda, um terceiro tipo de reação, denominada neurite isolada 8,9,10,11,15,19,20 que pode ocorrer tanto em pacientes classificados como paucibacilar (PB) ou multibacilar (MB) 9 .

No curso da doença crônica que é a hanseníase, cerca de 30-50\% dos pacientes podem desenvolver episódios reacionais $9,11,13,21$. Por isso, a vigilância em saúde enquanto "processo contínuo e sistemático de coleta, consolidação, análise e disseminação de dados sobre eventos relacionados à saúde” 22 (p. 48) apresentase como uma estratégia de subsídio ao planejamento e à implementação de políticas públicas para o efetivo manejo clínico e epidemiológico das reações hansênicas.

Nesse contexto, Rondônia priorizou a vigilância das reações com base nos resultados da pesquisa operacional 23, onde se evidenciou as fragilidades dos serviços de saúde no manejo clínico destas. Para a notificação e acompanhamento desses eventos 24 , desenvolveu-se um sistema de informação - Sistema de Estados Reacionais em Hanseníase (SisReação), pioneiro no país, de modo que os dados são fornecidos em tempo real, possibilitando análise e intervenções oportunas.

A identificação precoce destes eventos traz benefícios e melhoria da qualidade de vida dos pacientes devido à possibilidade de intervenção terapêutica imediata, evitando a evolução para maior gravidade e desenvolvimento de incapacidades físicas ${ }^{8}$. Ainda assim, no Brasil não existe sistema de informação de abrangência nacional que avalie esses eventos clínicos no itinerário terapêutico ou no período após o tratamento de PQT. Deste modo, entende-se como imprescindível priorizar a vigilância das reações hansênicas.

Neste estudo, objetivou-se analisar o tempo e os fatores associados à ocorrência da reação hansênica durante o tratamento de PQT e após o término da PQT, no Estado de Rondônia, entre os anos de 2008 e 2016.

\section{Método}

Estudo de coorte não concorrente dos casos notificados de hanseníase com ocorrência de reação durante e após o tratamento de PQT, registrados no SisReação, em Rondônia, entre os anos 2008 e 2016. A definição do período de estudo foi baseada na implantação da vigilância clínico-epidemiológica das reações hansênicas. Após esse período observou-se fragilidade na alimentação do SisReação, o que foi retomado, em 2019, com a implementação do sistema. 


\section{Fonte de dados}

Utilizaram-se os dados do Sistema de Informação de Agravos de Notificação (SINAN-hanseníase) para qualificar o desfecho da reação hansênica oriundo do SisReação. O SINAN é a principal ferramenta utilizada pela vigilância da hanseníase nos municípios, estados e Distrito Federal. Entretanto, para avaliar as reações hansênicas não se é possível por meio dele, uma vez que o campo de acompanhamento no sistema é insuficiente para caracterizar as reações. Além disso, perde-se a possibilidade de acompanhar as possíveis reações após o tratamento em razão do encerramento do caso no SINAN-hanseníase com a finalização da PQT.

Diante disso, Rondônia desenvolveu o SisReação que, desde 2007, notifica os casos de hanseníase com ocorrência de reação durante e após o tratamento de PQT, superando as limitações observadas no SINAN-hanseníase. O objetivo do SisReação é efetivar a vigilância das reações hansênicas como evento clínico epidemiológico de interesse para a saúde pública.

\section{População do estudo}

A população do estudo compreendeu 1.621 pessoas, composta pelos casos de hanseníase em reação durante o tratamento de $\mathrm{PQT}$ e após o término do tratamento notificados no SisReação, no período de 2008 a 2016, as quais preencheram os seguintes critérios de inclusão: modo de entrada caso novo, outras entradas, recidiva e outros reingressos notificados no SINAN-hanseníase do período de 2001 a 2016. Foram excluídos os casos notificados com tipo de saída duplicidade, transferências para outros estados ou país e erro de diagnóstico.

\section{Linkage entre o SINAN-hanseníase e a base do SisReação}

Para qualificar o desfecho reação hansênica, aplicou-se a técnica de relacionamento probabilístico entre os dois bancos, SINAN-hanseníase (2001 a 2016), SisReação (2008 a 2016), sendo este o arquivo de referência (segundo terminologia utilizada no programa ReckLink III) 25,26. Cabe pontuar que se expandiu o período do banco do SINAN-hanseníase para aumentar a sensibilidade na captura do maior número de pares possíveis. Utilizaram-se as variáveis condicionantes: "número da notificação" no SINAN-hanseníase, "nome do paciente", "data de nascimento" e "nome da mãe", no SINAN e SisReação. As bases utilizadas foram do Estado de Rondônia, obtidas junto à Agência Estadual de Vigilância em Saúde (AGEVISA), e garantida a confidencialidade dos dados.

\section{Tratamento dos dados}

Posteriormente à consolidação da base de dados do SINAN-hanseníase e do SisReação, caracterizaram-se as variáveis independentes à pessoa: sexo, raça/cor de pele, faixa etária, escolaridade, zona de residência, data do diagnóstico da hanseníase, esquema terapêutico, data do início do tratamento, forma clínica (classificação de Madrid), baciloscopia, grau de incapacidade física no diagnóstico, situação atual do paciente, tipo de reação, data do início do tratamento da reação, grau de incapacidade física na reação, conforme recomendações do Ministério da Saúde. A variável número de lesões de pele, campo do SINAN-hanseníase, que é preenchido pelo registro, do momento do diagnóstico, foi excluída das análises uma vez que se encontrou pacientes PB com mais de cinco lesões de pele. Ressalta-se que, ainda assim, para as análises, optou-se pela classificação operacional do momento da notificação da reação hansênica (SisReação), observadas as manifestações clínicas da reação com a classificação operacional.

\section{Análise estatística}

Os dados foram expressos como contagem absoluta e frequência relativa em porcentagens. Para o desfecho da temporalidade da reação, empregou-se a função de sobrevida de Kaplan-Meier, que usou como evento o aparecimento da primeira reação hansênica. Para o cálculo do tempo, em meses, 
decorrido até a reação hansênica, utilizou-se como referência a data do diagnóstico de hanseníase. Nessa análise, a precocidade do aparecimento da primeira reação hansênica foi comparada entre grupos $\mathrm{PB}$ e $\mathrm{MB}$, nos distintos períodos de ocorrência das reações, durante e após tratamento de PQT, bem como no período agregado (durante + após tratamento de PQT). Além disso, outros grupos foram criados para avaliar sua relação com a precocidade da reação hansênica nos mesmos períodos: sexo, faixa etária, raça/cor de pele, escolaridade, zona de residência, forma clínica, baciloscopia, grau de incapacidade física no diagnóstico. Para testar as diferenças entre os grupos quanto ao tempo até a ocorrência da primeira reação aplicou-se o teste de log-rank de Mantel-Cox.

Para identificar os fatores associados à ocorrência da reação (hazard ratio) foram construídos modelos de regressão de Cox univariada e multivariada em intervalo de 95\% de confiança (IC95\%). Variáveis com valores de $\mathrm{p}<0,2$ na análise univariada foram selecionadas para modelos multivariados. Então, foi realizado o método backward stepwise, permanecendo no modelo final aquelas que se mantiveram no valor de $\mathrm{p}<0,05$. As análises foram realizadas utilizando o software SPSS versão 23.0 para Macintosh (https://www.ibm.com/).

Nas análises dos modelos de função de sobrevida e de riscos proporcionais de Cox foram excluídas os ignorados e as categorias da raça/cor amarela e indígena pelo pequeno número de pacientes nestes grupos da população de estudo.

\section{Aspectos éticos}

O estudo foi aprovado pelo Comitê de Ética e Pesquisa da Universidade de Brasília sob parecer no 4.036.640, de 19 de maio de 2020, CAAE no 28800420.6.0000.0030, respeitando os aspectos éticos contidos no Decreto no 93.933/1987 e Resolução no 466/2012 do Conselho Nacional de Saúde.

\section{Resultados}

Após o processo de exclusão de 284 casos de hanseníase em reação em duplicidade e transferidos para outros estados ou países, resultou em 1.719 casos notificados no período de 2008 a 2016 no SisReação. No SINAN-hanseníase, no período de 2001 a 2016 foram excluídos 265 casos de hanseníase em duplicidade e transferidos para outros estados ou países, resultando em 18.190 casos. O pareamento probabilístico entre os bancos de dados sugeriu 1.621 (94,3\%) pares verdadeiros. As reações ocorreram durante o tratamento de PQT em 997 (61,5\%) e 624 (38,5\%) somente após o tratamento (Figura 1).

Dos 1.621, 1.468 (90,6\%) eram casos novos, 35 (2,1\%) outras entradas (entradas por transferências) 31 (1,9\%) outros reingressos (abandono, mudança de classificação operacional, 2o ciclo de esquema $\mathrm{MB})$ e 87 (5,4\%) recidivas.

As características sociodemográficas, clínicas e terapêuticas estão descritas na Tabela 1. Dos 1.621 pacientes avaliados em reação hansênica, 144 (8,9\%) eram PB e 1.477 (91,1\%) MB; a maioria da população de estudo era composta por homens, MB 927 (62,8\%), entre os quais 808 (54,7\%) tinham idade > 40 anos, 785 (53,2\%) eram de raça/cor de pele parda, 954 (64,6\%) com escolaridade no Ensino Fundamental e $962(65,1 \%)$ eram moradores da zona urbana. Na ocasião da reação, tanto nos PB quanto nos MB, a maior proporção dos pacientes estava em tratamento PQT.

Quanto às características clínicas e terapêuticas, 144 (100\%) dos pacientes PB eram da forma clínica tuberculoide e 1.019 (69\%) dos MB eram do grupo dimorfo. A baciloscopia (bacilos álcool-ácido resistentes - BAAR) é um exame complementar e foi realizado na maioria dos casos PB e MB, tendo o resultado negativo em 585 (39,6\%) entre os MB. No total de pacientes avaliados, as reações mais frequentes foram a neurite $808(49,8 \%)$, seguida da $R R=395(24,4 \%)$. O grau de incapacidade física (GIF) no diagnóstico da hanseníase foi avaliado em $97,2 \%$ e na reação $85,5 \%$.

Avaliaram-se as taxas de sobrevida, tempo, em meses, decorrido até o aparecimento do primeiro episódio de reação, tendo como referência a data do diagnóstico de hanseníase. A avaliação se deu comparando os grupos PB e MB nos períodos, durante o tratamento da PQT (997), após o tratamento (624) e no período agregado (durante a PQT + após tratamento - 1.621). A distribuição dos meses até o evento foi assimétrica, por isso utilizou-se a mediana do intervalo interquartil (IIQ) e não a média. 


\section{Figura 1}

Fluxograma do relacionamento das bases de dados dos Sistema Nacional de Agravos de Notificação (SINAN) e Sistema de Estados Reacionais em Hanseníase/Rondônia (SisReação/RO) quanto a população de estudo. Rondônia, Brasil, 2001 a 2016.

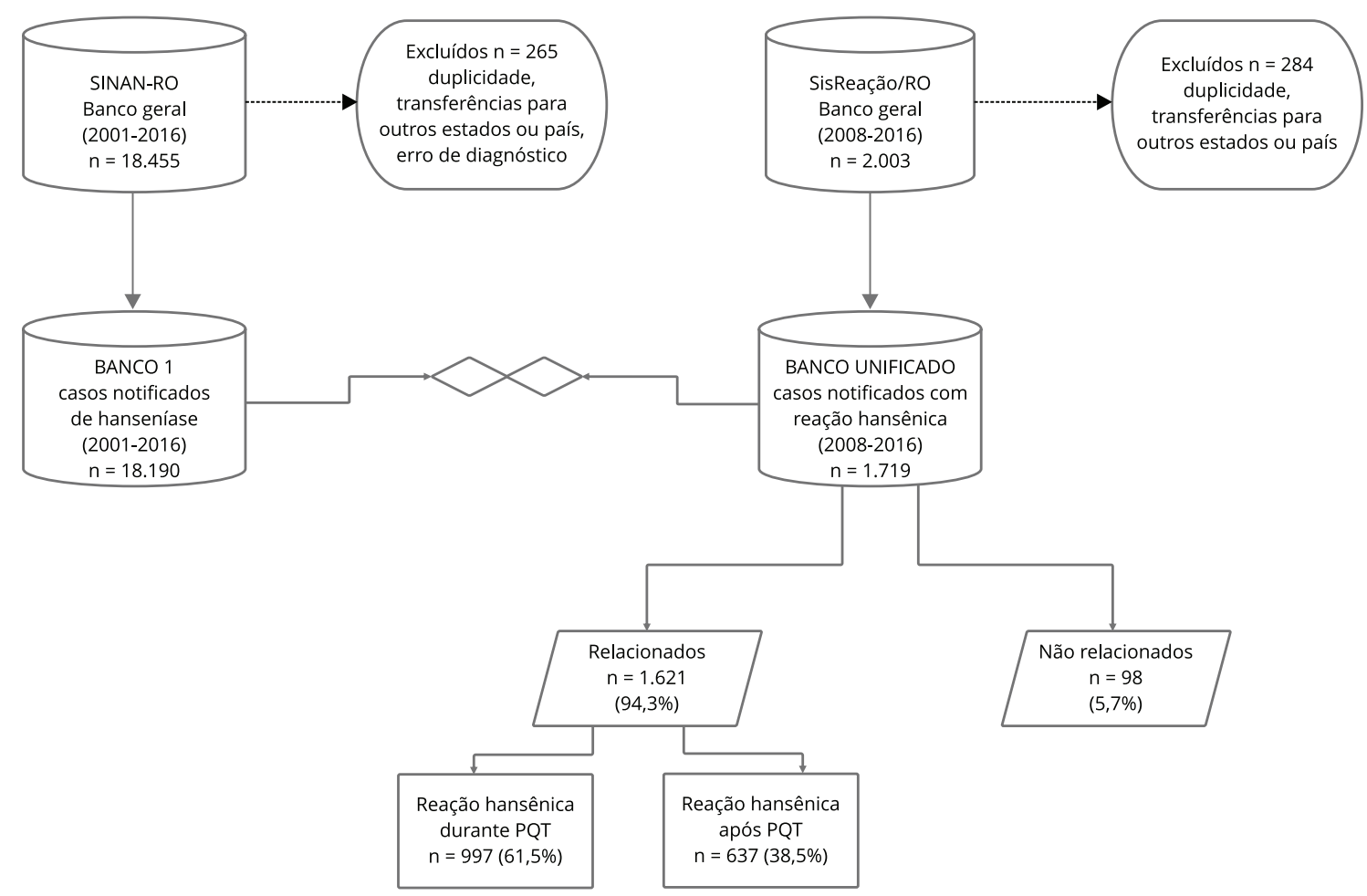

PQT: poliquimioterapia.

As seguintes variáveis associaram-se ao menor tempo de ocorrência da reação: sexo, classificação operacional e baciloscopia.

A Tabela 2 mostra que no período agregado, o sexo feminino manifestou o primeiro episódio reacional em sete meses após o diagnóstico da hanseníase, comparado ao sexo masculino (7,0 IIQ: 6,2-7,8 versus 9,0 IIQ: 7,8-10,2; valor de $\mathrm{p}=0,017)$; os pacientes $\mathrm{PB}$ tiveram o primeiro episódio em seis meses, comparados aos MB (6,0 IIQ: 4,6-7,4 versus 8,0 IIQ: 7,1-8,09; valor de $\mathrm{p}<0,001)$ e, aqueles pacientes com baciloscopia negativa apresentaram a reação em cinco meses após o diagnóstico (5,0 IIQ: 4,445,56; valor de $\mathrm{p}<0,001)$. Não houve diferenças estatisticamente significantes para faixa etária, raça/ cor de pele, escolaridade, zona de residência, forma clínica e GIF no diagnóstico.

Durante o tratamento de PQT, os pacientes PB tiveram a reação mais rápida, três meses após o diagnóstico da hanseníase, comparados aos $\mathrm{MB}$ (3,0 IIQ: 2,1- 3,8 versus 4,0 IIQ: 3,6-4,3; valor de $\mathrm{p}=0,002$ ) (Figura 2). Neste período, a única variável associada estatisticamente à precocidade da reação foi a classificação operacional.

No período após o tratamento, observou-se que a mediana em meses para a reação nos pacientes PB foi menor, de 22 meses, a partir do diagnóstico da hanseníase, em relação aos MB (22,0 IIQ: 16,127,8 versus 29,0 IIQ: 26,5-31,5; valor de $\mathrm{p}<$ 0,001) (Figura 3). As variáveis sexo, faixa etária, raça/ cor de pele, escolaridade, zona de residência, forma clínica, baciloscopia e GIF no diagnóstico não influenciaram a precocidade da reação hansênica. 


\section{Tabela 1}

Características sociodemográficas, clínicas e terapêuticas dos casos de hanseníase com reação. Rondônia, Brasil, 2008 a 2016.

\begin{tabular}{|c|c|c|}
\hline & \multicolumn{2}{|c|}{ Classificação operacional [n (\%)] } \\
\hline & Paucibacilar & Multibacilar \\
\hline Total & $144(8,9)$ & $1.477(91,1)$ \\
\hline \multicolumn{3}{|l|}{ Sexo } \\
\hline Masculino & $76(52,8)$ & $927(62,8)$ \\
\hline Feminino & $68(47,2)$ & $550(37,2)$ \\
\hline \multicolumn{3}{|l|}{ Faixa etária (anos) } \\
\hline$\leq 40$ & $54(37,5)$ & $668(45,3)$ \\
\hline$>40$ & $90(62,5)$ & $808(54,7)$ \\
\hline \multicolumn{3}{|l|}{ Raça/cor pele } \\
\hline Branca & $50(34,7)$ & $489(33,1)$ \\
\hline Preta & $10(6,9)$ & $136(9,2)$ \\
\hline Parda & $79(54,9)$ & $785(53,2)$ \\
\hline Amarela & $1(0,7)$ & $13(0,9)$ \\
\hline Indígena & $1(0,7)$ & $1(0,1)$ \\
\hline Ignorado & $7(4,9)$ & $48(3,2)$ \\
\hline \multicolumn{3}{|l|}{ Escolaridade } \\
\hline Analfabeto & $13(9,0)$ & $132(8,9)$ \\
\hline Ensino Fundamental & $95(66,0)$ & $954(64,6)$ \\
\hline Ensino Médio & $24(16,7)$ & $212(14,4)$ \\
\hline Ensino Superior & $1(0,7)$ & $32(2,2)$ \\
\hline \multicolumn{3}{|l|}{ Zona de residência } \\
\hline Urbana & $94(65,3)$ & $962(65,1)$ \\
\hline Rural & $41(28,5)$ & $428(29,0)$ \\
\hline Periurbana & $0(0,0)$ & $17(1,2)$ \\
\hline \multicolumn{3}{|l|}{ Situação atual do paciente } \\
\hline Em tratamento & $81(56,3)$ & $916(62,0)$ \\
\hline Em alta & $63(43,8)$ & $560(37,9)$ \\
\hline \multicolumn{3}{|l|}{ Forma clínica } \\
\hline Tuberculoide & $144(100,0)$ & $0(0,0)$ \\
\hline Dimorfa & $0(0,0)$ & $1.019(69,0)$ \\
\hline Virchowiana & $0(0,0)$ & $443(30,0)$ \\
\hline \multicolumn{3}{|l|}{ Baciloscopia } \\
\hline Positiva & $0(0,0)$ & $545(36,9)$ \\
\hline Negativa & $84(58,3)$ & $585(39,6)$ \\
\hline Não realizada & $60(41,7)$ & $347(23,5)$ \\
\hline \multicolumn{3}{|l|}{ Tipo de reação } \\
\hline Reação RR & $36(25,0)$ & $359(24,3)$ \\
\hline Neurite & $95(66,0)$ & $713(48,3)$ \\
\hline Reação RR + Neurite & $8(5,6)$ & $123(8,3)$ \\
\hline Reação ENH & $0(0,0)$ & $134(9,1)$ \\
\hline Reação ENH + Neurite & $4(2,8)$ & $104(7,0)$ \\
\hline Reação RR + ENH & $1(0,7)$ & $44(3,0)$ \\
\hline \multicolumn{3}{|c|}{ Incapacidade física no diagnóstico } \\
\hline Grau 0 & $82(56,9)$ & $788(53,4)$ \\
\hline Grau 1 & $36(25,0)$ & $475(32,2)$ \\
\hline Grau 2 & $23(16,0)$ & $171(11,6)$ \\
\hline Não avaliados & $3(2,1)$ & $43(2,8)$ \\
\hline
\end{tabular}

(continua) 


\begin{tabular}{lcc}
\hline & \multicolumn{2}{c}{$\begin{array}{c}\text { Classificação operacional [n (\%)] } \\
\text { Multibacilar }\end{array}$} \\
\hline Incapacidade física na reação & & \\
Grau 0 & $59(41,0)$ & $544(36,8)$ \\
Grau 1 & $44(30,6)$ & $486(32,9)$ \\
Grau 2 & $17(11,8)$ & $236(16,0)$ \\
Não avaliados & $24(16,6)$ & $211(14,3)$
\end{tabular}

ENH: eritema nodoso hansênico; RR: reação Tipo 1 ou reversa.

Fonte: Sistema Nacional de Agravos de Notificação; Sistema de Estados Reacionais em Hanseníase/Rondônia (SisReação/RO).

A Tabela 3 apresenta os resultados da análise univariada e multivariada por meio do modelo de regressão de Cox com os respectivos valores de hazard ratio (HR) e intervalo de 95\% de confiança (IC95\%), quanto aos fatores associados ao risco de ocorrência da reação hansênica. Os fatores que se associaram significativamente $(\mathrm{p}<0,05)$ foram: $\mathrm{PB}$ e baciloscopia negativa.

Constatou-se que no período agregado, os pacientes $\mathrm{PB}$ apresentaram risco $24 \%$ maior de reação do que os MB (HR = 1,244; IC95\%: 1,108-1,397; valor de p < 0,001) e aqueles com baciloscopia negativa aumentaram este risco em 40\% comparado à baciloscopia positiva (HR =1,407; IC95\%: 1,15-1,721; valor de $\mathrm{p}<0,001)$.

Durante e após o tratamento PQT, os PB tiveram 1,3 e 1,6 vezes maior risco à ocorrência da reação do que os MB (HR = 1,35; IC95\%: 1,090-1,673; valor de p = 0,007 e HR = 1,68; IC95\%: 1,111-2,541; valor de $\mathrm{p}=0,014$, respectivamente).

\section{Discussão}

Entre os participantes do estudo, as reações hansênicas foram mais frequentes entre os homens, na raça/cor de pele parda, $\mathrm{MB}$, do grupo dimorfo, dos tipos neurite e RR, durante o tratamento PQT. As principais características associadas à precocidade da reação foram classificação operacional, sexo e baciloscopia. Os pacientes PB e a baciloscopia negativa demonstraram-se com risco à ocorrência de reação em menor tempo.

A hanseníase incide em maior proporção entre homens 27,28,29,30. Nobre et al. 31 constataram que a chance de desenvolver hanseníase MB é duas vezes maior para homens do que para mulheres em todos os estados brasileiros. Admite-se maior frequência de reação hansênica em homens 11,20, respaldando os nossos resultados.

A influência do sexo é observada no processo saúde-doença-cuidado, sendo que os homens se tornam mais vulneráveis ao adoecimento e a formas mais graves, não apenas pelos seus hábitos de vida, mas também pela inadequação dos serviços de saúde na identificação e atendimento de suas necessidades específicas de saúde 32 .

A maior parte das pessoas observadas neste estudo era da raça/cor de pele negra (pretos e pardos), consoante ao perfil epidemiológico nacional da hanseníase 27,28,29,30. Há evidências de que esse grupo de brasileiros está associado a um aumento de $40 \%$ de risco de adoecimento por hanseníase, bem como a níveis mais altos de privação ${ }^{4}$, relacionada à desigualdade social 33 , desvelando os desafios que essas pessoas encontram para acessar os serviços de saúde e ao cuidado integral, situação imbricada historicamente a esta doença 4 . 


\section{Tabela 2}

Estimativas dos tempos até a reação hansênica por meio da análise de sobrevida de Kaplan-Meier, avaliando parâmetros sociodemográficos e clínicos nos períodos agregados, durante o tratamento de poliquimioterapia (PQT) e após o término do tratamento. Rondônia, Brasil, 2008 a 2016.

\begin{tabular}{|c|c|c|c|c|c|c|}
\hline & \multicolumn{2}{|c|}{ Períodos agregados [n (IC95\%)] } & \multicolumn{2}{|c|}{ Durante PQT [n (IC95\%)] } & \multicolumn{2}{|c|}{ Após PQT [n (IC95\%)] } \\
\hline & $\begin{array}{l}\text { Tempo até primeira } \\
\text { reação, em meses }\end{array}$ & Valor de $p$ & $\begin{array}{l}\text { Tempo até primeira } \\
\text { reação, em meses }\end{array}$ & Valor de $p$ & $\begin{array}{l}\text { Tempo até primeira } \\
\text { reação, em meses }\end{array}$ & Valor de $p$ \\
\hline Sexo & & 0,017 & & 0,143 & & 0,548 \\
\hline Masculino & $9,0(7,8-10,2)$ & & $4(3,5-4,4)$ & & $29(26,0-31,9)$ & \\
\hline Feminino & $7,0(6,2-7,8)$ & & $4(3,5-4,4)$ & & $28(23,9-32,0)$ & \\
\hline Faixa etária (anos) & & 0,182 & & 0,307 & & 0,201 \\
\hline$\leq 40$ & $8(7,0-8,9)$ & & $4(3,5-4,4)$ & & $29(26,1-31,8)$ & \\
\hline$>40$ & $8(6,7-9,2)$ & & $4(3,5-4,4)$ & & $28(24,4-31,5)$ & \\
\hline Raça/cor & & 0,258 & & 0,335 & & 0,085 \\
\hline Branco & $7(5,8-8,1)$ & & $4(3,4-4,5)$ & & $31(27,8-34,1)$ & \\
\hline Preto & $12(7,8-16,1)$ & & $4(2,8-5,1)$ & & $26(20,1-31,8)$ & \\
\hline Pardo & $7(6,1-7,8)$ & & $4(3,5-4,4)$ & & $26(23,1-28,8)$ & \\
\hline Escolaridade & & 0,521 & & 0,693 & & 0,296 \\
\hline Analfabeto & $9(5,7-12,2)$ & & $3(2,2-3,7)$ & & $25(14,8-35,1)$ & \\
\hline Ensino Fundamental & $8(7,0-8,9)$ & & $4(3,6-4,3)$ & & $30(27,3-32,6)$ & \\
\hline Ensino Médio & $7(5,3-8,6)$ & & $4(3,3-4,6)$ & & $23(16,6-29,3)$ & \\
\hline Ensino Superior & $6(4,1-7,8)$ & & $5(3,4-6,5)$ & & $32(11,8-52,1)$ & \\
\hline Zona & & 0,176 & & 0,167 & & 0,257 \\
\hline Urbana & $7(6,1-7,8)$ & & $4(3,6-4,3)$ & & $26(22,4-29,5)$ & \\
\hline Rural & $7(5,7-8,2)$ & & $3(2,3-3,6)$ & & $31(26,3-35,6)$ & \\
\hline Periurbana & $5(0,0-11,7)$ & & $3(1,8-4,1)$ & & $19(8,2-29,7)$ & \\
\hline Classificação operacional & & $<0,001$ & & 0,002 & & $<0,001$ \\
\hline Paucibacilar & $6(4,6-7,4)$ & & $3(2,1-3,8)$ & & $22(16,1-27,8)$ & \\
\hline Multibacilar & $8(7,1-8,9)$ & & $4(3,6-4,3)$ & & $29(26,5-31,5)$ & \\
\hline Forma clínica & & 0,392 & & 0,112 & & 0,710 \\
\hline Tuberculoide & $7(5,5-8,4)$ & & $3(2,1-3,9)$ & & $23(15,8-30,1)$ & \\
\hline Dimorfa & $7(6,2-7,7)$ & & $4(3,5-4,4)$ & & $29(26,1-31,8)$ & \\
\hline Virchowiana & $10(7,7-12,2)$ & & $4(3,3-4,6)$ & & $29(24,8-33,1)$ & \\
\hline Baciloscopia & & $<0,001$ & & 0,284 & & 0,099 \\
\hline Positiva & $7(5,9-8,0)$ & & $4(3,3-4,6)$ & & $24(21,3-26,6)$ & \\
\hline Negativa & $5(4,4-5,5)$ & & $4(3,5-4,4)$ & & $21(18,9-23,0)$ & \\
\hline Grau de incapacidade & & 0,159 & & 0,149 & & 0,497 \\
\hline \multicolumn{7}{|l|}{ física no diagnóstico } \\
\hline Grau 0 & $8(6,8-9,1)$ & & $4(3,5-4,4)$ & & $29(25,6-32,3)$ & \\
\hline Grau 1 & $7(5,9-8,0)$ & & $4(3,4-4,5)$ & & $28(24,2-31,7)$ & \\
\hline Grau 2 & $7(4,5-9,4)$ & & $3(2,1-3,9)$ & & $25(18,4-31,5)$ & \\
\hline
\end{tabular}

IC95\%: intervalo de 95\% de confiança.

Fonte: Sistema Nacional de Agravos de Notificação; Sistema de Estados Reacionais em Hanseníase/Rondônia (SisReação/RO).

Nota: dados expressos como estimativa da mediana do tempo até a reação no intervalo interquartil; valores de p determinados pela estatística de log-rank de Cox-Mantel. 


\section{Figura 2}

Curva de Kaplan-Meier mostrando taxas de sobrevida até ocorrência da reação hansênica segundo classificação operacional no período durante tratamento de poliquimioterapia (PQT). Rondônia, Brasil, 2008 a 2016.

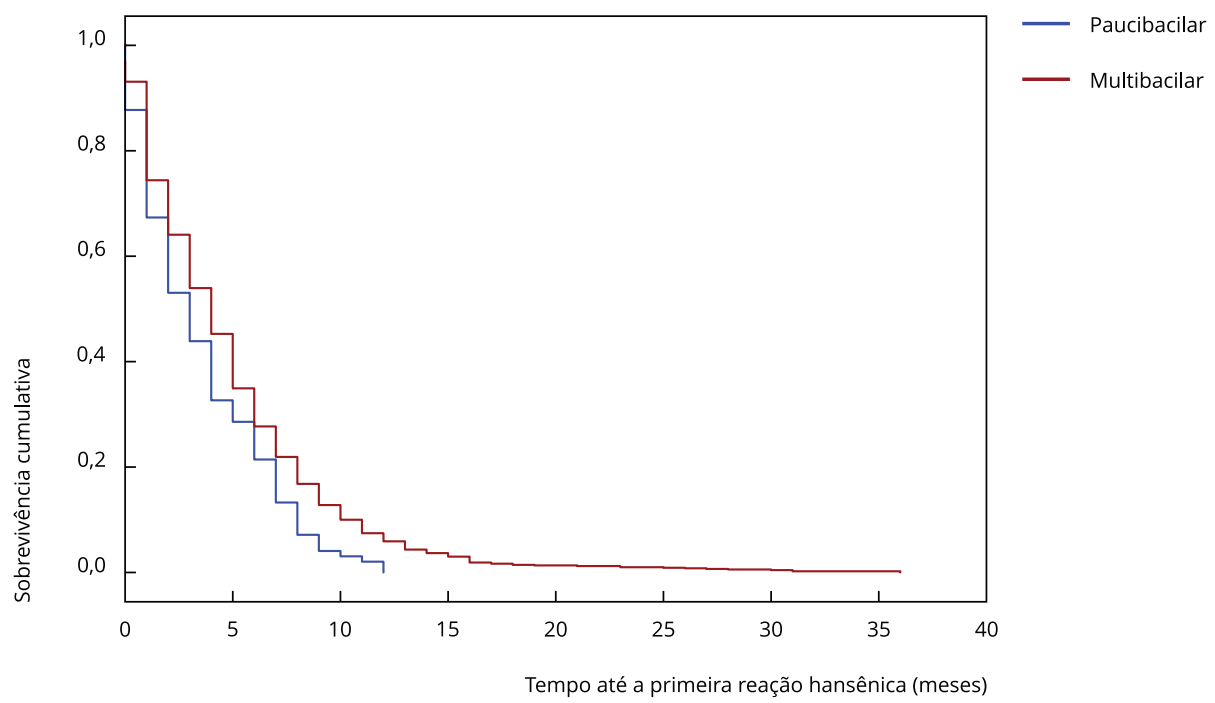

Figura 3

Curva de Kaplan-Meier mostrando taxas de sobrevida até ocorrência da reação hansênica segundo classificação operacional no período após o término do tratamento. Rondônia, Brasil, 2008 a 2016.






\section{Tabela 3}

Análise da regressão de Cox univariada e em modelos multivariados para tempo até reação hansênica. Rondônia, Brasil, 2008 a 2016.

\begin{tabular}{|c|c|c|c|c|}
\hline & \multicolumn{4}{|c|}{ Tempo até primeira reação hansênica } \\
\hline & \multicolumn{2}{|c|}{ Análise univariada } & \multicolumn{2}{|c|}{ Análise multivariada * } \\
\hline & HR (IC95\%) & Valor de $p$ & HR (IC95\%) & Valor de $p$ \\
\hline \multicolumn{5}{|c|}{ Período agregado } \\
\hline \multicolumn{5}{|l|}{ Sexo } \\
\hline Masculino & 1,000 & & & \\
\hline Feminino & $1,125(1,018-1,244)$ & 0,021 & & \\
\hline \multicolumn{5}{|c|}{ Faixa etária (anos) } \\
\hline$\leq 40$ & 1,000 & & & \\
\hline$>40$ & $0,937(0,850-1,034)$ & 0,197 & & \\
\hline \multicolumn{5}{|l|}{ Raça/Cor } \\
\hline Branca & 1,000 & & & \\
\hline Preto & $1,003(0,835-1,204)$ & 0,977 & & \\
\hline Pardos & $1,086(0,975-1,209)$ & 0,136 & & \\
\hline \multicolumn{5}{|l|}{ Zona } \\
\hline Urbana & 1,000 & & & \\
\hline Rural & $0,939(0,842-1,047)$ & 0,259 & & \\
\hline Periurbana & $1,301(0,806-2,102)$ & 0,282 & & \\
\hline \multicolumn{5}{|c|}{ Classificação operacional } \\
\hline Multibacilar & 1,000 & & & \\
\hline Paucibacilar & $1,437(1,209-1,707)$ & $<0,001$ & $1,244(1,108-1,397)$ & $<0,001$ \\
\hline \multicolumn{5}{|l|}{ Baciloscopia } \\
\hline Positiva & 1,000 & & & \\
\hline Negativa & $1,233(1,100-1,381)$ & $<0,001$ & $1,407(1,150-1,721)$ & $<0,001$ \\
\hline \multicolumn{5}{|c|}{ Período do tratamento } \\
\hline \multicolumn{5}{|l|}{ Sexo } \\
\hline Masculino & 1 & & & \\
\hline Feminino & $1,09(0,959-1,237)$ & 0,186 & & \\
\hline \multicolumn{5}{|c|}{ Faixa etária (anos) } \\
\hline$\leq 40$ & 1,000 & & & \\
\hline$>40$ & $1,060(0,936-1,201)$ & 0,358 & & \\
\hline \multicolumn{5}{|l|}{ Raça/Cor } \\
\hline Branca & 1,000 & & & \\
\hline Preto & $0,85(0,667-1,082)$ & 0,187 & & \\
\hline Pardos & $0,977(0,853-1,12)$ & 0,742 & & \\
\hline \multicolumn{5}{|l|}{ Zona } \\
\hline Urbana & 1,000 & & & \\
\hline Rural & $1,092(0,951-1,255)$ & 0,213 & & \\
\hline Periurbana & $1,224(0,691-2,168)$ & 0,488 & & \\
\hline \multicolumn{5}{|c|}{ Classificação operacional } \\
\hline Multibacilar & 1,000 & & & \\
\hline Paucibacilar & $1,352(1,096-1,667)$ & 0,005 & $1,35(1,090-1,673)$ & 0,007 \\
\hline \multicolumn{5}{|l|}{ Baciloscopia } \\
\hline Positiva & 1,000 & & & \\
\hline Negativa & $1,069(0,933-1,225)$ & 0,335 & & \\
\hline
\end{tabular}

(continua) 


\begin{tabular}{|c|c|c|c|c|}
\hline & \multicolumn{4}{|c|}{ Tempo até primeira reação hansênica } \\
\hline & \multicolumn{2}{|c|}{ Análise univariada } & \multicolumn{2}{|c|}{ Análise multivariada * } \\
\hline & HR (IC95\%) & Valor de $p$ & HR (IC95\%) & Valor de $p$ \\
\hline \multicolumn{5}{|c|}{ Período pós-alta } \\
\hline \multicolumn{5}{|l|}{ Sexo } \\
\hline Masculino & 1,000 & & & \\
\hline Feminino & $1,051(0,891-1,241)$ & 0,556 & & \\
\hline \multicolumn{5}{|c|}{ Faixa etária (anos) } \\
\hline$\leq 40$ & 1,000 & & & \\
\hline$>40$ & $0,902(0,768-1,059)$ & 0,208 & & \\
\hline \multicolumn{5}{|l|}{ Raça/Cor } \\
\hline Branca & 1,000 & & & \\
\hline Preto & $1,281(0,965-1,701)$ & 0,087 & & \\
\hline Pardos & $1,185(0,993-1,413)$ & 0,059 & & \\
\hline \multicolumn{5}{|l|}{ Zona } \\
\hline Urbana & 1,000 & & & \\
\hline Rural & $0,917(0,768-1,093)$ & 0,333 & & \\
\hline Periurbana & $1,466(0,606-3,546)$ & 0,396 & & \\
\hline \multicolumn{5}{|c|}{ Classificação operacional } \\
\hline Multibacilar & 1,000 & & & \\
\hline Paucibacilar & $1,755(1,293-2,382)$ & $<0,001$ & $1,68(1,111-2,541)$ & 0,014 \\
\hline \multicolumn{5}{|l|}{ Baciloscopia } \\
\hline Positiva & 1,000 & & & \\
\hline Negativa & $1,185(0,963-1,459)$ & 0,109 & & \\
\hline
\end{tabular}

IC95\%: intervalo de 95\% de confiança ; HR: hazard ratio.

Fonte: Sistema Nacional de Agravos de Notificação; Sistema de Estados Reacionais em Hanseníase/Rondônia (SisReação/RO).

* Foi usado o método backward stepwise.

A maioria dos pacientes desenvolveram reação durante a poliquimioterapia, confirmando as evidências que apontam para maior proporção das reações nesse período 20,33,34,35. Há autores que relatam cerca de 10 a 50\% 11,21, outros 74\% 36, principalmente nas formas multibacilares. No Brasil, o estudo em pacientes multibacilares tratados com esquema único U-MDT (MB - 6 meses) e esquema R-MDT (MB - 12 meses), a frequência de reação não mostrou diferença estatisticamente significante entre os dois grupos, sendo que mais da metade dos pacientes não desenvolveram reação em seis meses de tratamento, em ambos grupos avaliados 2 .

As reações RR acometem entre 10 a 33\% dos pacientes com hanseníase 11, geralmente do grupo dimorfo (D), dimorfo-tuberculoide (DT) e dimorfo virchowiano (DV) 9,10,37 e surgem durante o tratamento ou após o primeiro ano do término da PQT 11. Outros autores observaram que o primeiro surto reacional apareceu com maior frequência quando já iniciado o tratamento específico em mais da metade dos pacientes. Além disso, afirmam que a neurite isolada esteve presente na grande maioria dos pacientes avaliados 8,19,38,39, corroborando com os achados deste estudo. A neurite isolada é aquela que se apresenta com sintomas ou sinais neurais, sem manifestações cutâneas de RR ou ENH 8,9,40. Assim, a neurite constitui-se em quadro grave, que requer intervenção e conduta médica imediata, para prevenir o estabelecimento de incapacidades físicas 10,41.

A neurite durante as reações se manifesta com dor espontânea ou à palpação, acompanhada de alteração da função neural 13,41. Nery et al. (1993, apud Alves et al. 9, p. 190) "acharam uma frequência de neurite isolada em pacientes dimorfos de 57,1\%, quando comparada com outros tipos reacionais". Corroborando, Lockwood \& Saunderson 39 afirmam que a inflamação perineural mediada por células T, é o principal processo patológico na lesão dos nervos na hanseníase e, portanto, são classificados como casos limítrofes (grupo dimorfo), perfil que está compatível à população desse estudo. Vale ressal- 
tar que Rondônia é um dos poucos estados do Brasil que incorporou o tratamento cirúrgico para neurite 42,43,44. Embora a literatura sustente os resultados aqui apresentados, considera-se desenvolver estudo sobre a prevalência de neurite para desvendar sua alta frequência.

Do ponto de vista do cuidado, a ocorrência das reações no curso do tratamento propicia oportunidade para diagnóstico e intervenção em tempo oportuno no nível primário de atenção. Em consenso a isso, Freitas et al. 33 (p. 6601) abordam que a "Estratégia de Saúde da Família pode auxiliar na detecção precoce dos casos, o que é de suma importância para a prevenção das sequelas e incapacidades advindas dessa doença”. Para tanto, são necessárias ações como orientações aos pacientes quanto o possível surgimento das reações 35 , capacitação das equipes de saúde com abordagem para reconhecer e tratar as reações, disponibilidade dos antirreacionais na atenção primária à saúde (APS) e, quando necessário, retaguarda de unidades de maior complexidade de atenção.

Apesar de evidências científicas direcionarem para uma maior proporção das reações durante a PQT, não se pode perder de vista o acompanhamento dos casos que apresentam reações após o tratamento, sobretudo pelo potencial de piora das deformidades físicas neste período 39,45 . Reforça-se que estudos apontam para a ocorrência de reação após o tratamento entre 15\% a 23\% dos casos 46,47, corroborando os achados deste estudo em que cerca de um terço apresentou reação neste período particular. Deste modo, sugere-se a implementação da vigilância das reações e das incapacidades físicas durante o seguimento do tratamento e após a alta.

Destaca-se ainda no pós alta, a necessidade de diferenciar reação e recidiva que, por vezes, sua clínica se confunde 20 , haja vista que aqueles que sofriam com episódios reacionais tiveram mais possibilidades de retratamento por suspeita de recidiva da hanseníase 11.

O desenvolvimento de reações após o tratamento é frequentemente definido pelos pacientes como sintomas da doença, interferindo na sua qualidade de vida 2. Dessa maneira, recomenda-se aos coordenadores dos programas de hanseníase promoverem fóruns, educação permanente e sobretudo, matriciamento à APS abordando as reações e as diferenças entre recidiva, já que o apoio matricial em saúde objetiva assegurar retaguarda especializada à equipe e profissionais encarregados da atenção aos problemas de saúde 48 .

A precocidade da reação hansênica nos pacientes $\mathrm{PB}$ poderia ser explicado em razão da boa imunidade celular que é desencadeada pelo início do tratamento PQT, ao instalar o processo inflamatório agudo secundário a liberação de antígenos com a quebra dos bacilos com reações de hipersensibilidade 49, mas também, as reações podem estar associadas à resposta terapêutica e à capacidade de resposta imunológica do paciente. As reações desencadeadas nos pacientes $\mathrm{PB}$ remetem à RR, geralmente acompanhada de comprometimento neural periférico ou mesmo, a neurite isolada 10 .

Surpreendentemente, a baciloscopia negativa influenciou à precocidade da reação, resultado que pode ser elucidado pelas características clínicas dos participantes do estudo, em que os pacientes $\mathrm{PB}$ possuem baixa carga bacilar, portanto, sempre terão baciloscopia negativa; e entre os $\mathrm{MB}$, prevaleceu o grupo dimorfo, dos quais a maior parte teve a baciloscopia negativa, o que sugere a possibilidade de estar associada à resposta imune celular (do tipo Th1). Esse achado difere dos estudos que correlacionam reação e índice baciloscópico (IB), em que a frequência das reações está associada direta e proporcionalmente à positividade do IB 1,19,20,50, numa proporção de quanto maior o IB 18,50, mais frequente e mais precoce a reação nos $\mathrm{MB}$, indicando uma associação entre IB alto e um padrão de reação ENL 21, contudo, a taxa de RR foi significativamente maior entre os pacientes com $\mathrm{IB}<31,21$. Observou-se também que no estudo caso-e-controle "a reação após a alta foi estatisticamente associada à carga bacilar através da positividade do teste sorológico nesse período” 20 (p. 67).

A população deste estudo tem características clínicas que poderiam assemelhar-se segundo classificação de Ridley \& Jopling (bordeline-tuberculoide - BT, bordeline-bordeline - BB) ou na classificação Madrid (dimorfo-tuberculoide - DT, e dimorfo-dimorfo - DD), que levariam em consideração a imunidade dentro de um espectro de resistência do hospedeiro, no qual a imunidade celular é inversamente proporcional à carga bacilar 12,49. À vista disso, estão no espectro do polo da boa resposta imunidade celular 1,2,49 e, portanto, é no grupo dimorfo que incide a maior frequência de reações reversas, respaldando os resultados para a grande maioria dos pacientes em reação dos tipos neurite e RR.

Segundo Opromolla 51 (p. 54), "tanto na classificação de Madrid como na de Ridley er Jopling se admite a instabilidade dos dimorfos, e isso seria devido a $R R$ a que esses casos estão sujeitos. Seja como for, e adotando 
a nomenclatura dada por Ridley er Jopling, os BT seriam aqueles casos semelhantes aos do polo tuberculoide", da classificação de Madrid.

Assim, tem-se como uma das hipóteses a insegurança dos profissionais na definição da forma clínica e na decisão de tratar os pacientes por mais tempo, classificando-os como $\mathrm{MB}$, o que pode ser justificado pela impossibilidade de se ter, em campo, todas as ferramentas para a classificação tão precisa 2,9. A despeito das limitações do diagnóstico, que ainda é essencialmente clínico-epidemiológico, a fragilidade dos vínculos empregatícios na APS, sobretudo do médico 52 , produz perda da força de trabalho qualificada nas ações de controle da hanseníase. Similarmente, pode-se atribuir à fragilidade na identificação do tipo de reação, sobretudo da neurite isolada que exige dos profissionais acurácia na palpação de nervos periféricos associada às queixas e alteração da função neural, particularmente em comparação a uma avaliação neurológica inicial ou de seguimento. São desafios impostos aos profissionais da APS, os quais, para superá-los, carecem de prioridade da gestão para oferta de educação permanente, com vista no desenvolvimento humano e institucional.

O sexo feminino associou-se à precocidade da reação no período agregado, contudo, Saunderson et al. 37 (p. 313) revelaram que "ser mulher foi um fator de risco para o aparecimento tardio do primeiro episódio de RR". São poucos os estudos clínicos que versam sobre reações hansênicas, e, portanto, observa-se como campo aberto para novas investigações que elucidem as relações entre as reações e sexo.

Do ponto de vista social, a reação hansênica é um fator que afeta a qualidade de vida das mulheres, particularmente pelo uso dos corticosteroides, indicados para o tratamento das reações 53 . Apesar dos grandes benefícios terapêuticos, os corticosteroides podem causar efeitos adversos, em especial nas terapias prolongadas (por mais de 30 dias) ou em altas doses 54 , como são os esquemas recomendados para as reações hansênicas. Entre esses efeitos adversos, cita-se a síndrome de Cushing iatrogênica que é provocada pelo uso excessivo ou prolongado da prednisona, em que as mulheres têm três vezes mais chances de desenvolver a síndrome do que os homens 55 , a qual gera mudanças no seu corpo, comprometendo sua autoestima e sua condição feminina 53.

Neste estudo os pacientes PB demostraram risco à ocorrência de reação em menor tempo em qualquer período (agregado, durante e após o tratamento de PQT) e a baciloscopia negativa apresentou risco à ocorrência de reação no período agregado, ratificando os achados em que os casos classificados limítrofes (DT, DD) 8 são fatores de risco à ocorrência de RR 37.

A identificação de fatores que predispõem à reação durante e após o tratamento de PQT, entende-se como imperativo para prevenir incapacidades físicas. Embora tenha progresso na compreensão da imunopatologia das reações hansênicas 56, não existem exames laboratoriais capazes de prevê-las 1,20,35, havendo interesse para determinar marcadores clínicos ou laboratoriais que identifiquem pacientes com aumento do risco para o desenvolvimento das reações 36 .

As fragilidades do SINAN no registro do número de lesões de pele foi um limitador para abordar a correlação de lesões de pele especificamente com reação e, sobretudo, com a precocidade dos episódios. Entretanto, um estudo realizado no Brasil evidenciou que a distribuição das lesões dermatológicas se torna útil por indicar o potencial de RR ou ENH entre os pacientes MB 21, além de que associou-se ao agravamento da deformidade física após o tratamento com PQT 45. Por isso, considera-se primordial o zelo no registro das lesões de pele, visto que existem evidências quanto à associação entre a presença de mais de cinco lesões com a ocorrência de reação 21,57. Destaca-se a importância de registros com qualidade, precisos e confiáveis, para que venham sustentar e direcionar ações resolutivas à tomada de decisão.

A limitação de dados secundários e estudos retrospectivos podem causar imprecisão de registro de informação, como observado nas variáveis classificação operacional, número de lesões de pele e forma clínica ou quando não se tem registro, a exemplo da dor neuropática. Neste sentido, deve-se ter cautela na generalização dos achados. Outrossim, somam-se às limitações do estudo a indisponibilidade, nos sistemas, do IB e da classificação de Ridley \& Jopling; a Organização Mundial da Saúde (OMS) padronizou para uso nos serviços a classificação de Madrid, a qual adota critérios de polaridade, baseados nas características clínicas, bacteriológicas e histológicas da hanseníase 38 .

Em que pese as limitações expostas, esse trabalho explorou um cenário ainda pouco estudado no Brasil, registrando um panorama das reações hansênicas, estabelecendo uma linha de base para novas investigações. Além disso, esses achados podem colaborar com o planejamento de estratégias e fomento de novas políticas públicas no contexto da saúde dessa população. 
Salienta-se neste estudo alguns pontos fortes, como a operacionalização da vigilância das reações hansênicas como evento clínico epidemiológico, por meio de um sistema de informação capaz de acompanhar e avaliar as reações, de modo a subsidiar o plano de atenção longitudinal e integral às pessoas acometidas pela hanseníase, além de permitir o planejamento dos medicamentos antireacionais e de insumos para a prevenção e reabilitação de incapacidades físicas.

\section{Considerações finais}

A identificação de fatores associados à precocidade das reações hansênicas pode contribuir para vigilância desses eventos clínicos. Dessa forma, as evidências permitem recomendar a priorização de ações de vigilância das reações durante e após o tratamento PQT, como medidas de prevenção de incapacidades físicas e de melhoria na qualidade de vida das pessoas com hanseníase.

À vista disso, enfatiza-se a prioridade dos coordenadores estaduais e municipais para assegurar o suporte técnico e farmacêutico necessário à APS de modo a identificar precocemente e instituir a terapêutica adequada às reações hansênicas, bem como estabelecer os pontos de atenção na rede do Sistema Único de Saúde (SUS) para referenciar, quando necessário.

Ademais, o conjunto de resultados observados neste estudo apontam para a necessidade de implementar tecnologias para o diagnóstico precoce da hanseníase com vistas a minimizar a ocorrência de reações e debelar as incapacidades físicas decorrentes da detecção tardia da doença.

\section{Colaboradores}

C. R. F. Coriolano e M. N. Sanchez contribuíram na concepção do estudo, análise e interpretação de dados, elaboração do texto, aprovação final e são responsáveis por todos os aspectos do estudo. W. A. Freitas Neto contribuiu na elaboração do texto, análise e interpretação dos dados. G. O. Penna contribuiu na análise e interpretação de dados, revisão do texto e revisão final do conteúdo intelectual.

\section{Informações adicionais}

ORCID: Carmelita Ribeiro Filha Coriolano (00000002-9616-1217); Walter Ataalpa de Freitas Neto (0000-0001-9776-5509); Gerson Oliveira Penna (0000-0001-8967-536X); Mauro Niskier Sanchez (0000-0002-0472-1804)

\section{Referências}

1. Hungria EM, Bührer-Sékula $S$, Oliveira RM, Aderaldo LC, Pontes AA, Cruz R, et al. Leprosy reactions: the predictive value of Mycobacterium leprae-specific serology evaluated in a Brazilian cohort of leprosy patients (U-MDT/CT-BR). PLoS Negl Trop Dis 2017; 11:e0005396.

2. Penna GO, Bührer-Sékula S, Kerr LRS, Stefani MMA, Rodrigues LC, Araújo MG, et al. Uniform multidrug therapy for leprosy patients in Brazil (U-MDT/CT-BR): results of an open label, randomized and controlled clinical trial, among multibacillary patinetes. PLoS Negl Trop Dis 2017; 11:e0005725.

3. Souza EA, Boigny RN, Oliveira HX, Oliveira MLW, Heukelbach J, Alencar CH, et al. Tendências e padrões espaço-temporais da mortalidade relacionada à hanseníase no Estado da Bahia, Nordeste do Brasil, 1999-2014. Cad Saúde Colet (Rio J.) 2018; 26:191-202.

4. Nery JS, Ramond A, Pescarini JM, Alves A Strina A, Ichihara MY, et al. Socioeconomic determinants of leprosy new case detection in the 100 Million Brazilian Cohort: a population-based linkage study. Lancet Glob Health 2019; 7:e1226-36. 
5. Sanchez MN, Nery JS, Pescarini JM, Mendes AA, Ichihara MY, Teixeira CSS, et al. Physical disabilities caused by leprosy in 100 million cohort in Brazil. BMC Infect Dis 2021; 21:290.

6. World Health Organization. Global leprosy (Hansen disease) update, 2019: time to stepup prevention initiatives. Wkly Epidemiol Rec 2020; 95:417-40.

7. Departamento de Doenças de Condições Crônicas e Infecções Sexualmente Transmissíveis, Secretaria de Vigilância em Saúde, Ministério da Saúde. Estratégia nacional para enfrentamento da hanseníase 2019-2022. Brasília: Ministério da Saúde; 2021.

8. Nery JADC, Sales AM, Illarramendi X, Duppre NC, Jardim MR, Machado AM. Contribution to diagnosis and management of reactional states: a practical approach. An Bras Dermatol 2006; 81:367-75.

9. Alves ED, Ferreira TL, Ferreira IN, organizadores. Hanseníase: avanços e desafios. Brasília: Universidade de Brasília; 2014.

10. Foss NT. Episódios reacionais na hanseníase. Medicina (Ribeirão Preto) 2003; 36:453.

11. Teixeira MAG, Silveira VM, França ER. Characteristics of leprosy reactions in paucibacillary and multibacillary individuals attended at two reference centers in Recife, Pernambuco. Rev Soc Bras Med Trop 2010; 43:287-92.

12. Bernardes Goulart IM, Penna GO, Cunha G. Immunopathology of leprosy: the complexity of the mechanisms of host immune response to Mycobacterium leprae. Rev Soc Bras Med Trop 2002; 35:365-75.

13. Saunderson P. Como reconhecer e tratar reações hansênicas. 2a Ed. Belo Horizonte: The International Federation of Anti-Leprosy Associations; 2007.

14. Scollard DM, Smith T, Bhoopat L, Theetranont C, Rangdaeng S, Morens DM. Epidemiologic characteristics of leprosy reactions. Int J Lepr Other Mycobact Dis 1994; 62:559-67.

15. Penna MLF, Buhrer-Sékula S, Pontes MADA, Cruz R, Gonçalves HDS, Penna GO. Primary results of clinical trial for uniform multidrug therapy for leprosy patients in Brazil (UMDT/CT-BR): reactions frequency in multibacillary patients. Lepr Rev 2012; 83:308-19.

16. Suchonwanit P, Triamchaisri S, Wittayakornrerk S, Rattanakaemakorn P. Leprosy reaction in Thai population: a 20-year retrospective study. Dermatol Res Pract 2015; 2015:253154.

17. Walker SL. Leprosy reactions. In: Scollard D, Gillis T, editors. International textbook of leprosy. s.1.: Papyros Designs; 2002. p. 3-29.

18. Regional Office for South-East Asia, World Health Organization. Leprosy: management of reactions and prevention of disabilities. Technical guidance. New Delhi: World Health Organization; 2020.

19. Gonçalves SD, Sampaio RF, Antunes CMF. Ocorrência de neurite em pacientes com hanseníase: análise de sobrevida e fatores preditivos TT. Rev Soc Bras Med Trop 2008; 41:464-9.
20. Brito MFM, Ximenes RAA, Gallo MEN, Bührer-Sékula S. Associação entre reação hansênica após alta e a carga bacilar avaliada utilizando sorologia anti PGL-I e baciloscopia. Rev Soc Bras Med Trop 2008; 41 Suppl 2:67-72.

21. Nery JAC, Vieira LMM, De Matos HJ, Gallo MEN, Sarno EN. Reactional states in multibacillary hansen disease patients during multidrug therapy. Rev Inst Med Trop São Paulo 1998; 40:363-70.

22. Ministério da Saúde. Portaria no 1.378, de 09 de julho de 2013. Regulamenta as responsabilidades e define diretrizes para execução e financiamento das ações de Vigilância em Saúde pela União, Estados, Distrito Federal e Municípios, relativos ao Sistema Nacional de Vigilância em Saúde e Sistema Nacional de Vigilância Sanitária. Diário Oficial da União 2013; 9 jul.

23. Oliveira CR, Alencar MJ, Sena Neto SA, Santana SC, Ramos Jr. AN. Fatores que influenciaram a inadequação do diagnóstico e do acompanhamento dos estados reacionais em hanseníase no Estado de Rondônia, Brasil. Hansen Int 2007; 32:185-96.

24. Secretaria de Estado da Saúde de Rondônia. Portaria Estadual no 82, de 18 de março de 2014. Diário Oficial do Estado de Rondônia 2014; 20 mar.

25. Coeli CM, Pinheiro RS, Camargo Jr. KR. Conquistas e desafios para o emprego das técnicas de record linkage na pesquisa e avaliação em saúde no Brasil. Epidemiol Serv Saúde 2015; 24:795-802.

26. Pinheiro RS, Camargo Júnior KR, Coeli CM. Relacionamento de bases de dados em saúde. Cad Saúde Colet (Rio J.) 2006; 14:195-6.

27. Secretaria de Vigilância em Saúde, Ministério da Saúde. Hanseníase. Boletim Epidemiológico 2018; 49(4). http://portalarquivos2.saude. gov.br/images/pdf/2018/janeiro/31/2018004-Hanseniase-publicacao.pdf.

28. Secretaria de Vigilância em Saúde, Ministério da Saúde. Hanseníase. Hanseníase. Boletim Epidemiológico 2020; 51. https://www.saude. gov.br/images/pdf/2020/July/14/Boletimepidemiologico-SVS-28-v2.pdf.

29. Secretaria de Vigilância em Saúde, Ministério da Saúde. Hanseníase. Boletim Epidemiológico 2020; Número Especial. https://portalarqui vos2.saude.gov.br/images/pdf/2020/janei ro/31/Boletim-hanseniase-2020-web.pdf.

30. Secretaria de Vigilância em Saúde, Ministério da Saúde. Hanseníase. Boletim Epidemiológico 2021; Número Especial. http://www.aids.gov. br/pt-br/pub/2021/boletim-epidemiologicohanseniase-2021.

31. Nobre ML, Illarramendi X, Dupnik KM, Hacker MA, Nery JAC, Jerônimo SMB, et al. Multibacillary leprosy by population groups in Brazil: lessons from an observational study. PLoS Negl Trop Dis 2017; 11:e0005364. 
32. Souza EA, Boigny RN, Ferreira AF, Alencar $\mathrm{CH}$, Oliveira MLW, Ramos Jr. AN. Vulnerabilidade programática no controle da hanseníase: padrões na perspectiva de gênero no Estado da Bahia, Brasil. Cad Saúde Pública 2018; 34:e00196216.

33. Freitas LC, Costa JA, Oliveira ACS, Miranda JO, Rodrigues KO, Lopes WB, et al. Fatores associados ao tipo de reação hansênica: um estudo caso controle. Brazilian Journal of Development 2020; 6:6599-609.

34. Raffe SF, Thapa M, Khadge S, Tamang K, Hagge D, Lockwood DNJ. Diagnosis and treatment of leprosy reactions in integrated services - the patients' perspective in Nepal. PLoS Negl Trop Dis 2013; 7:e2089.

35. Queiroz TA, Carvalho FPB, Simpson CA, Fernandes ACL, Figueirêdo DLA, Knackfuss MI. Perfil clínico e epidemiológico de pacientes em reação hansênica. Rev Gaúcha Enferm 2015; 36(esp):185-91.

36. Sociedade Brasileira de Hansenologia, Sociedade Brasileira de Dermatologia. Hanseníase: episódios reacionais. s.l.: Associação Médica Brasileira; Conselho Federal de Medicina; 2003.

37. Saunderson P, Gebre S, Byass P. Reversal reactions in the skin lesions of AMFES patients: Incidence and risk factors. Lepr Rev 2000; 71:309-17.

38. Antonio JR, Soubhia RMC, Paschoa VD, Oliveira GB, Rossi NCP, Maciel MG, et al. Avaliação epidemiológica dos estados reacionais e incapacidades físicas em pacientes com hanseníase na cidade de São José do Rio Preto. Arq Ciênc Saúde 2011; 18:9-14.

39. Lockwood DN, Saunderson PR. Nerve damage in leprosy: a continuing challenge to scientists, clinicians and service providers. Int Health 2012; 4:77-85.

40. Porichha D, Mukherjee A, Ramu G. Neural pathology in leprosy during treatment and surveillance. Lepr Rev 2004; 75:233-41.

41. Pimentel MIF, Nery JAC, Borges E, Rolo R, Sarno EN. Silent neuritis in multibacillary leprosy evaluated through the development of disabilities before, during and after multidrug therapy. An Bras Dermatol 2004; 79:169-79.

42. Alencar MJF, Barbosa JC, Oliveira C, Ramos Jr. AN, Schreuder PAM, Amaral RCG, et al. Satisfação de indivíduos atingidos pela hanseníase a respeito de neurólise no estado de Rondônia. Cad Saúde Colet (Rio J.) 2008; 16:205-16.

43. De Oliveira CR, Freitas De Alencar MDJ, Sena Neto SAD, Lehman LF, Schreuder PAM. Impairments and Hansen's disease control in Rondônia State, Amazon region of Brazil. Lepr Rev 2003; 74:337-48.

44. Amaral RCG. Avaliação sensitiva e motora de pacientes com neurite hansênica submetidos à neurolósie no Estado de Rondonia, no período 2000 a 2003 [Dissertação de Mestrado]. Brasília: Universidade de Brasília; 2006.
45. Sales AM, Campos DP, Hacker MA, Nery JAC, Düppre NC, Rangel E, et al. Progression of leprosy disability after discharge: is multidrug therapy enough? Trop Med Int Health 2013; 18:1145-53

46. Silva SF, Griep RH. Leprosy reaction in patients of health centers from the Planning Area 3.2. of Rio de Janeiro Municipality. Hansenol Int 2007; 32:155-62.

47. Rodrigues ALP, Almeida AP, Rodrigues BF, Pinheiro CA, Borges DS, Mendonça MLH, et al. Ocorrência de reações em pacientes pós alta por cura de hanseníase: subsídios para implementação de um programa de atenção específica. Hansen Int 2000; 25:7-16.

48. Campos GWS, Domitti AC. Apoio matricial e equipe de referência: uma metodologia para gestão do trabalho interdisciplinar em saúde. Cad Saúde Pública 2007; 23:399-407.

49. Mendonça VA, Costa RD, De Melo GEBA, Antunes CM, Teixeira AL. Imunologia da hanseníase. An Bras Dermatol 2008; 83:343-50.

50. Saunderson P, Gebre S, Byass P. ENL reactions in the multibacillary cases of the AMFES cohort in central Ethiopia: incidence and risk factors. Lepr Rev 2000; 71:318-24.

51. Opromolla DVA. Manifestações clínicas e reações. In: Opromolla DVA, editor. Noções de hansenologia. Bauru: Centro de Estudos Dr. Reynaldo Quagliato; 2000. p. 51-8.

52. Pierantoni CR, Vianna CMM, França T, Magnago C, Rodrigues MPS. Rotatividade da força de trabalho médica no Brasil. Saúde Debate 2015; 39:637-47.

53. Loose JTT, Diniz SN, Batista EC, Carlotto MSC, Cunha DFFD. Qualidade de vida em mulheres com episódios reacionais hansênicos em uso de prednisona no município de Rolim de Moura -RO. Revista Ciência e Saúde On-line $2017 ; 2: 1-10$.

54. Departamento de Vigilância Epidemiológica, Secretaria de Vigilância em Saúde, Ministério da Saúde. Orientações para uso: corticosteroides em hanseníase. Brasília: Editora do Ministério da Saúde; 2010.

55. National Institute of Diabetes and Digestive and Kidney Diseases. Cushing's Syndrome https://www.niddk.nih.gov/healthinformation/endocrine-diseases/cushingssyndrome?dkrd=hispt0295\#1 (acessado em 22/Mai/2021).

56. Fava V, Orlova M, Cobat A, Alcaïs A, Mira M, Schurr E. Genetics of leprosy reactions: an overview. Mem Inst Oswaldo Cruz 2012; 107 Suppl I:132-42.

57. Ventura KG. Estudo das reacões hansênicas durante a poliquimioterapia em unidades de saúde da região metropolitana da Grande Vitória [Dissertação de Mestrado]. Vitória: Universidade Federal do Espírito Santo; 2006. 


\section{Abstract}

The clinical management of leprosy patients poses a specific challenge, namely lepra reactions. This non-concurrent cohort study aimed to analyze the timing of the first lepra reaction during and after polychemotherapy (PCT) and associated factors. A total of 1,621 patients were assessed ( $P B$ $=8.9 \%$ and $M B=91.1 \%$ ) from 2008 to 2016 , reported to the System of Reaction States in Leprosy (SisReação/RO) database. Reactions occurred predominantly during PCT (997; 61.5\%) and less frequently only after PCT $(624 ; 38.5 \%)$. Earliness of the reaction after diagnosis was analyzed with Kaplan-Meier survival curves, with comparison between the PB and MB groups using the MantelCox log-rank test. Univariate and multivariate Cox regression models were constructed to identify factors associated with occurrence of lepra reactions (hazard ratio) and the corresponding 95\%CI. The multivariate model included variables with $p$-values $<0.20$ in the univariate analysis. $P B$ patients developed reactions earlier than $M B$ patients. Other characteristics were associated with earlier reactions: female gender and negative smear microscopy. In the aggregate period (during and after $P C T$ ), $P B$ presented $24 \%$ higher risk of lepra reaction than $M B$ patients, and negative smear microscopy increased this risk by $40 \%$ compared to positive smear microscopy. During and after PCT, $P B$ presented 1.3 and 1.6 times the risk, respectively, of reactions when compared to $M B$ patients. We thus recommend prioritizing surveillance of lepra reactions during and after PCT as measures to prevent physical disabilities and to improve quality of life for persons with leprosy.

Leprosy; Epidemiologic Surveillance; Survival Analysis

\section{Resumen}

La gestión clínica de pacientes con hanseniasis presenta un desafío particular que son las reacciones. El objetivo de este estudio de cohorte no concurrente fue analizar el tiempo y factores asociados a la ocurrencia de la primera reacción durante y tras el tratamiento de la poliquimioterapia $(P Q T)$. Se evaluó a 1621 pacientes $(P B=$ $8,9 \%$ y $M B=91,1 \%$ ) de 2008 a 2016, notificados en el Sistema de Estados Reaccionarios en Lepra (SisReação/RO). Prevaleció la ocurrencia durante la $P Q T=997(61,5 \%)$, y $624(38,5 \%)$ solamente tras la PQT. La precocidad de la reacción a partir del diagnóstico se analizó mediante curvas de supervivencia de Kaplan-Meier y se compararon entre los grupos PB y MB, usando el test de logrank de Mantel-Cox; asimismo, se construyeron modelos de regresión univariada y multivariada de Cox para identificar los factores asociados con la ocurrencia de la reacción (cociente de riesgos) y los correspondientes IC95\%. En el modelo multivariado se incluyeron las variables con valores de $p<0,2$ en el análisis univariado. Los PB desarrollaron una reacción de forma más precoz que los MB. Otras características se asociaron a la reacción en menor tiempo: sexo femenino y baciloscopia negativa. En el período agregado (durante y tras $P Q T$ ), los $P B$ presentaron un riesgo un $24 \%$ mayor de reacción que los multibacilares y la baciloscopia negativa aumentó este riesgo en un 40\%, comparado con la baciloscopia positiva. Durante $y$ tras el tratamiento $P Q T$, los $P B$ presentaron 1,3 y 1,6 veces el riesgo de ocurrencia de la reacción de los pacientes MB. De esa forma, recomendamos priorizar acciones de vigilancia para reacciones hansénicas durante y tras $P Q T$, como medidas de prevención de discapacidades físicas, así como de mejoría en la calidad de vida de personas afectadas por la hanseniasis.

Lepra; Vigilancia Epidemiológica; Análisis de Supervivencia
Recebido em 23/Fev/2021

Versão final reapresentada em 23/Mai/2021

Aprovado em 25/Jun/2021 